\title{
REPEAT PROSTATE BIOPSIES FOLLOWING DIAGNOSES OF PROSTATE INTRAEPITHELIAL NEOPLASIA AND ATYPICAL SMALL GLAND PROLIFERATION
}

\author{
KATIA R. LEITE, CRISTINA A. MITTELDORF, LUIZ H. CAMARA-LOPES \\ Laboratory of Surgical and Molecular Pathology, Syrian-Lebanese Hospital, Sao Paulo, SP, Brazil
}

\begin{abstract}
Objective: To assess the incidence of diagnosis of high-grade intraepithelial neoplasia or prostate intraepithelial neoplasia (PIN), and atypical small gland proliferation (ASAP) at a uropathology reference center. To assess the indexes and findings on repeat biopsies.

Materials and Methods: Diagnoses of PIN, ASAP or PIN + ASAP established between January 1, 2001 and December 31, 2003 were searched in our database. We studied repeat biopsies performed up to August 31, 2004.

Results: Of 1420 biopsies, ASAP was diagnosed in $26(1.8 \%)$ patients, PIN in $142(10 \%)$ and PIN + ASAP in $40(2.8 \%)$. Repeat biopsies were performed in 98 patients, $16(61.5 \%)$ with ASAP, $53(37.3 \%)$ with PIN and $29(72.5 \%)$ with PIN + ASAP. Carcinoma was diagnosed in 7 cases $(43.8 \%)$ following a diagnosis of ASAP, $12(41.4 \%)$ of PIN + ASAP and $7(13.2 \%)$ of PIN. The mean interval between repeat biopsies was 299.6 days. There was no difference between groups where cancer was or was not diagnosed on repeat biopsy in relation to age and serum PSA levels.

Conclusion: Despite explicit recommendations of repeat biopsy on pathology reports and the high incidence of adenocarcinoma on repeat biopsy, re-intervention rates following a diagnosis of PIN, ASAP, PIN + ASAP are low in our setting. The diagnosis that most frequently led to repeat biopsy was PIN + ASAP. Adenocarcinoma was most often diagnosed after the initial diagnosis of ASAP.
\end{abstract}

Key words: prostate; needle biopsies; prostatic intraepithelial neoplasia; adenocarcinoma Int Braz J Urol. 2005; 31: 131-6

\section{INTRODUCTION}

The finding of pre-malignant lesions or suspected adenocarcinoma in prostate biopsies has become commonplace after the increase in the number of patients undergoing the procedure and the increased number of fragments obtained. High-grade intraepithelial neoplasia (PIN) is the only admittedly pre-malignant lesion of the prostate preceding or accompanying invasive carcinoma, and is identified in approximately $9 \%$ of biopsies, ranging from 0.7 to $20 \%$ (1). The detection of cancer on repeat biopsy is approximately $30 \%$, and can reach $75 \%$ when more than 3 fragments contain the lesion on the first biopsy (2). It is known that the finding of adenocarcinoma on repeat biopsy is independent of prostate specific antigen (PSA) serum levels, and even patients with a slight or zero increase in PSA have a 50\% risk of presenting adenocarcinoma on a repeat biopsy performed within a period of 3 years (3). The second condition is the finding of atypical glands whose morphological criteria are insufficient for the definitive diagnosis of carcinoma. Known as ASAP (atypical small acinar proliferation), it is not a distinct en- 
tity but a tumor that is marginally reached by the biopsy or one of the many conditions mimicking cancer. Its incidence ranges from 1.5 to $5.5 \%$, with cancer being diagnosed in up to $60 \%$ of repeat biopsies (4).

This study aims to assess the incidence of PIN and ASAP in our population, as well as the index and histological findings on repeat biopsies.

\section{MATERIALS AND METHODS}

We reviewed the reports from 1420 prostate biopsies that had been analyzed between January 1 , 2001 and December 31, 2003 at the Laboratory of Surgical and Molecular Pathology of the Syrian-Lebanese Hospital, and findings were categorized into carcinoma, benign, PIN and ASAP. The same pathologist examined all biopsies. Four hundred and fifty seven biopsies $(32.2 \%)$ were sent for review and the remaining 963 were processed as usual. The mean and median age of the patients was 65 and 66 years respectively, and the mean and median number of biopsied fragments was 12 . We investigated repeat prostate biopsies in patients who had been diagnosed with PIN, ASAP or both as of August 31, 2004.

\section{RESULTS}

Findings from 1420 biopsies are in Table-1. In 208 patients $(14.7 \%)$ the diagnosis was PIN, ASAP or PIN + ASAP. PIN was diagnosed in 142 patients
(10\%), ASAP in $26(1.8 \%)$ and PIN + ASAP in 40 (2.8\%). Repeat biopsies were performed on 98 patients, representing $47.1 \%$ of the diagnoses of PIN, ASAP or PIN + ASAP. The mean interval for repeat biopsy was 299.6 days, ranging from 1 to 1136 days.

The number of repeat biopsies, findings and intervals are in Table-2. Information concerning PSA was available for only 111 of the 208 patients with ASAP, PIN or PIN + ASAP, with 56 undergoing repeat biopsy.

Mean serum PSA values of patients undergoing repeat biopsy in related to the primary diagnosis and the repeat biopsy in Table-3. In 71 patients $(72.5 \%)$ a single repeat biopsy was performed, 20 $(20.5 \%)$ received 2 repeat biopsies, $5(5.1 \%)$ received 3 repeat biopsies, and only 2 patients underwent 4 repeat biopsies. The diagnosis of cancer was established in 26 patients $(26.5 \%)$ undergoing repeat biopsy. The initial diagnosis that led to the most cancer diagnoses in repeat biopsy was ASAP, with cancer being found in 7 patients (43.8\%), all on the first repeat biopsy. The diagnosis of PIN + ASAP followed, with repeat biopsy diagnosing cancer in 12 patients (41.4\%), 8 on the first repeat biopsy and 4 on the second intervention. Following the diagnosis of PIN, 7 cases $(13.2 \%)$ of adenocarcinoma were identified, 5 on the first repeat biopsy, 1 on the third and 1 on the fourth intervention.

The number of fragments affected by PIN, ASAP and PIN + ASAP, is not correlated with the

Table 1 - Findings in 1420 biopsies performed over 3 year period.

\begin{tabular}{lccc}
\hline Diagnosis & N $(\%)$ & Mean and Median Age (Range) & $\begin{array}{c}\text { Mean and Median Biopsied } \\
\text { Fragments (Range) }\end{array}$ \\
\hline Adenocarcinoma & $843(59.4)$ & 66.0 & 11.0 \\
\multirow{2}{*}{ ASAP } & $26(1.8)$ & $67.0(32-94)$ & $12.0(1-31)$ \\
& & 65.6 & 9.1 \\
PIN + ASAP & $40(2.8)$ & $67.5(49-76)$ & $9.5(1-21)$ \\
& & 65.0 & 14.0 \\
PIN & \multirow{2}{*}{$(10.0)$} & $67.0(49-78)$ & $14.0(2-24)$ \\
& & 64.0 & 14.0 \\
Benign & $369(26.0)$ & $64.0(47-84)$ & $14.0(2-32)$ \\
& & 63.1 & 13.6 \\
& & $63.0(43-86)$ & $13.0(3-28)$ \\
\hline
\end{tabular}


Table 2 - Repeat biopsies performed in 98 patients. Number, diagnosis and interval for repeat biopsies.

\begin{tabular}{|c|c|c|c|}
\hline Primary Diagnosis & Repeat Biopsies N (\%) & Diagnoses on Repeat Biopsy N (\%) & Mean Interval in Days (Range) \\
\hline ASAP & $16(61.5)$ & $\begin{array}{l}\text { Adenocarcinoma - } 7 \text { (43.8) } \\
\text { ASAP - } 1(6.2) \\
\text { PIN+ASAP - } 1(6.2) \\
\text { PIN - } 2(12.5) \\
\text { Benign - } 5(31.3)\end{array}$ & $119.5(1-374)$ \\
\hline $\mathrm{PIN}+\mathrm{ASAP}$ & $29(72.5)$ & $\begin{array}{l}\text { Adenocarcinoma - } 12(41.4) \\
\text { ASAP - } 6(20.7) \\
\text { PIN+ASAP - } 3(10.3) \\
\text { PIN - } 6(20.7) \\
\text { Benign - } 2(6.9)\end{array}$ & $\begin{array}{l}\text { 1st - } 200(14-795) \\
\text { 2nd - } 456(116-839) \\
3 r d-555(337-664)\end{array}$ \\
\hline PIN & $53(37.3)$ & $\begin{array}{l}\text { Adenocarcinoma - } 7 \text { (13.2) } \\
\text { ASAP - } 4 \text { (7.5) } \\
\text { PIN+ASAP - } 1(1.9) \\
\text { PIN - } 22(41.5) \\
\text { Benign - } 19(35.8)\end{array}$ & $\begin{array}{l}1 \text { st - } 256(21-937) \\
\text { 2nd }-553(197-1136) \\
\text { 3rd - } 525(328-704) \\
4 \text { th }-854(711-998)\end{array}$ \\
\hline
\end{tabular}

Table 3 - Serum PSA levels from 56 patients undergoing repeat biopsy. Primary diagnosis, diagnosis on repeat biopsy and mean PSA.

\begin{tabular}{llcc}
\hline Primary Diagnosis & Diagnosis on Repeat Biopsy & N & $\begin{array}{c}\text { PSA ng/mL } \\
\text { Mean (SD) - Median }\end{array}$ \\
\hline \multirow{2}{*}{ ASAP } & Adenocarcinoma & 3 & $5.7(4.8)-4.7$ \\
& PIN, ASAP, PIN + ASAP & 2 & $6.6(1.8)-6.6$ \\
& Benign & 3 & $10.2(2.3)-11$ \\
PIN + ASAP & Adenocarcinoma & 8 & $9.2(5.8)-8.1$ \\
& PIN, ASAP, PIN + ASAP & 6 & $6.8(1.6)-7.1$ \\
& Benign & 1 & 7.7 \\
PIN & Adenocarcinoma & 4 & $9.5(2.8)-9.4$ \\
& PIN, ASAP, PIN + ASAP & 16 & $6.2(2.5)-5.8$ \\
& Benign & 13 & $9.0(4.9)-8$ \\
\hline
\end{tabular}

final diagnosis of adenocarcinoma (Table-4). The mean and median value for Gleason histological grade of carcinomas found on repeat biopsy was 6 , and the mean number of fragments affected by the tumor was 3.3.

Of the 26 patients diagnosed with cancer, we examined the specimen of radical prostatectomy from
6 patients. None of the tumors was considered clinically insignificant; the Gleason histological grade was 6 in 3 patients, 7 in one and 8 in 2 . Tumor volume ranged from 1 to $16.4 \mathrm{cc}$, mean and median of 11.7 and $8.8 \mathrm{cc}$. Three patients were staged as pT2c, 2 pT3b and 1 pT3a (TNM 2002). 
Table 4 - Mean number of fragments affected by PIN, ASAP, PIN+ASAP lesions, in relation to the final diagnosis.

\begin{tabular}{llc}
\hline Primary Diagnosis & Diagnosis on Repeat Biopsy & Mean (SD) \\
\hline ASAP & Adenocarcinoma & $1.1(0.4)$ \\
& PIN, ASAP, PIN + ASAP & $1.0(0)$ \\
& Benign & $1.4(0.5)$ \\
PIN + ASAP & Adenocarcinoma & $4(3.8)$ \\
& PIN, ASAP, PIN + ASAP & $5.7(3.2)$ \\
& Benign & $1.7(1.2)$ \\
PIN & Adenocarcinoma & $2.5(0.6)$ \\
& PIN, ASAP, PIN + ASAP & $6.3(4.7)$ \\
& Benign & $4.5(3.0)$ \\
\hline
\end{tabular}

\section{COMMENTS}

Data presented in this paper are important because they derive from a reference uropathology department which conducts a large number of biopsy analyses every year, and can be seen as an indicator of the management adopted by the urologist when facing a diagnosis of PIN and ASAP. The numbers we found are also important since they are based on extended biopsies rather than restricted to sextants where the mean and median number of fragments was12, including biopsies that can be regarded as presenting saturation with 32 fragments. The incidence of PIN was $10 \%$, ASAP $1.8 \%$ and PIN plus ASAP was identified in $2.8 \%$ of 1420 biopsies analyzed over the 3-year period.

Though we explicitly suggest a repeat biopsy in our report on a routine basis for all cases containing ASAP, either alone or associated with PIN, we observed that $38.5 \%$ of patients diagnosed with ASAP did not undergo repeat biopsy, which occurred in $27.5 \%$ of the 40 cases diagnosed with PIN + ASAP. These numbers differ from the ones recently published, and they reflect the practice of urologists who are fellows at the American Oncology Association, where $98 \%$ of them requested a repeat biopsy when confronted with a diagnosis of ASAP (5). However, they are similar to studies by Fadare et al., who report the incidence of ASAP in $2.8 \%$ of cases, repeat biopsy at $67 \%$ and diagnosis of adenocarcinoma at $38 \%$ (6). Three other similar studies report rates for repeat biopsy of between $35 \%$ and 57\% (7-9).

The term ASAP was originally used by Iczkowski et al. (7), and it refers to minute tumor foci, a small lesion that disappears in other sectioning levels, or the absence of categorical cytological criteria for establishing the diagnosis of carcinoma. It is not consensually used and, in fact, it represents a marginally biopsied tumor or one of the many benign lesions that mimic cancer (10). Some authors use the term "atypical gland focus" in order to refer to suspected acinar structures containing no definitive morphologic parameters for diagnosing carcinoma. The incidence of this diagnosis is $5 \%$ and adenocarcinoma detection on the repeat biopsy is approximately 50\% (11).

In our samples of ASAP, all cancer diagnoses were established on the first repeat biopsy, with a mean interval of 119.5 days, with the near totality of reassessments being performed within the first year. In the presence of 2 lesions (PIN + ASAP), two repeat biopsies were required in one third of the patients for establishing the definitive diagnosis of cancer in a mean interval of 456 days, though extending up to 3 years.

The repeat biopsy strategy is extremely important on such occasions and the pathologist should always communicate the location of the fragment containing ASAP. The diagnosis of cancer is most 
probable in the sextant where ASAP was diagnosed, therefore a higher number of fragments should be removed from this region as well as from the adjacent sextants, in addition to fragments representative of other prostate regions (12).

Interestingly we observed that in cases diagnosed as ASAP or PIN + ASAP in our study, serum PSA levels were unable to predict the presence of cancer on the repeat biopsy. In cases of ASAP, mean and median PSA values were lower in individuals whose repeat biopsy showed adenocarcinoma when compared with those with suspected or benign diagnosis. However, other authors have found 2 times higher PSA levels in patients with a subsequent diagnosis of cancer $(13,14)$.

Among our patients whose cancer was diagnosed after the first diagnosis of ASAP, we examined the specimen from the radical prostatectomy in 4 cases. The Gleason grade was $8(4+4)$ in 2 patients, and $6(3+3)$ in another 2 . The mean tumor volume was $7.6 \mathrm{cc}$ ranging from 2.2 to $16.4 \mathrm{cc}$. Staging was pT2c in 2 cases and pT3b in 2, thus no tumor could be considered clinically insignificant. One study has recently shown that among 21 patients undergoing radical prostatectomy following diagnosis of ASAP, infiltrative cancer was identified in $100 \%$ (15). However, the study presents a significant bias concerning patient selection for surgery, where serum PSA levels and changes in digital rectal examinations were taken into account. A more judicious analysis of this study leads to the same cancer rates as other reports in the literature, around $47 \%$. We emphasize that the diagnosis of ASAP must be followed by a repeat biopsy, and it should not be treated as a cancer case until the definitive histological diagnosis is obtained.

In relation to a diagnosis of PIN, we observed a rate that is similar to the current literature, which describes a mean incidence of $9 \%$, ranging from 4 to $16 \%$ (1). Cancer detection on the repeat biopsy has been described in approximately $30 \%$ of cases, a higher rate than our 13\% (3). Most of our patients underwent one repeat biopsy, $21 \%$ underwent 2 repeat biopsies, $3.7 \%$ underwent 3 repeat biopsies and $3.7 \%$ underwent 4 repeat biopsies. Cancer was diagnosed on the first repeat biopsy in the majority of patients, with one case in the third and another in the fourth biopsy. Mean interval until repeat biopsy was 256 days, more than twice the cases of ASAP. The fourth biopsy was performed up to 3 years later. Our methodology analyzed the occurrence of repeat biopsies over a period of up to 6 months after the inclusion of the last patient diagnosed with PIN, ASAP or both. This fact can be responsible for the lower indexes of repeat biopsy in our patients, as well for the lower number of cases diagnosed with cancer in cases of PIN.

The strategy for repeat biopsy in patients diagnosed with PIN is different from the one used with ASAP patients. The biopsy should be performed randomly, since in approximately $40 \%$ of the cases the infiltrative tumor will be present in the lobe contralateral to the one where PIN was found (16). We must remember that PIN is an intraepithelial pre-malignant lesion, which accompanies or precedes invasive cancer and, differently from ASAP, does not represent a marginally biopsied tumor (1).

The number of fragments involved by PIN is reportedly correlated to findings on follow-up (2), however we did not confirm such data. During follow-up, the mean number of fragments with PIN in patients diagnosed with cancer was 2.5 and in those with benign or suspected diagnosis it was 5.7.

Similarly to the literature (3), we observed that mean serum PSA levels did not differ between the 2 groups either.

While examining the surgical specimen from a single patient after a diagnosis of cancer following PIN, the Gleason histological grade was $7(3+4)$, the volume was $9 \mathrm{cc}$ and the stage was pT3a, and similarly, clinically significant.

We have concluded that in our setting, despite the diagnosis of ASAP alone or accompanied by PIN having led to more than $70 \%$ of repeat biopsies, the indexes are lower than the ideal since we were able to diagnose cancer on repeat biopsy in more than $40 \%$ of patients, and all patients undergoing surgery had significant tumors.

\section{REFERENCES}

1. Bostwick DG, Qian J: High-grade prostatic intraepithelial neoplasia. Mod Pathol. 2004; 17: 360-79. 
2. Kronz JD, Allan CH, Shaikh AA, Epstein JI: Predicting cancer following a diagnosis of high-grade prostatic intraepithelial neoplasia on needle biopsy: data on men with more than one follow-up biopsy. Am J Surg Pathol. 2001; 25: 1079-85.

3. Lefkowitz GK, Taneja SS, Brown J, Melamed J, Lepor $\mathrm{H}$ : Follow up interval prostate biopsy 3 years after diagnosis of high grade prostatic intraepithelial neoplasia is associated with high likelihood of prostate cancer, independent of change in prostate specific antigen levels. J Urol. 2002; 168: 1415-8.

4. Epstein JI, Potter SR: The pathological interpretation and significance of prostate needle biopsy findings: implications and current controversies. J Urol. 2001; 166: 402-10.

5. Rubin MA, Bismar TA, Curtis S, Montie JE: Prostate needle biopsy reporting: how are the surgical members of the society of urologic oncology using pathology reports to guide treatment of prostate cancer patients? Am J Surg Pathol. 2004; 28: 946-52.

6. Fadare O, Wang S, Mariappan MR: Practice patterns of clinicians following isolated diagnoses if atypical small acinar proliferation on prostate biopsy specimens. Arch Pathol Lab Med. 2004; 128: 557-60.

7. Ickzowski KA, MacLennan GT, Bostwick DG: Atypical small acinar proliferation suspicious for malignancy in prostate needle biopsies: clinical significance in 33 cases. Am J Surg Pathol. 1997; 21: 1489-95.

8. Renshaw AA, Santis WF, Richie JP: Clinicopathological characteristics of prostatic adenocarcinoma in men with atypical prostate needle biopsies. J Urol. 1998; 159: 2018-21; discussion 2022.
9. Iczkoeski KA, Chen HM, Yang XJ, Beach RA: Prostate cancer diagnosed after initial biopsy with small acinar proliferation suspicious for malignancy is similar to cancer found on initial biopsy. Urology. 2002; 60: 851-4.

10. Srigley JR: Benign mimickers of prostatic adenocarcinoma. Mod Pathol. 2004; 17: 328-48.

11. Epstein JI: Diagnosis and reporting of limited adenocarcinoma of the prostate on needle biopsy. Mod Pathol. 2004; 17: 307-15.

12. Allen EA, Kahane H, Epstein JI: Repeat biopsy strategies for men with atypical diagnoses on initial prostate needle biopsy. Urology. 1998; 52: 803-7.

13. Alvarez-Alvarez C, Silva EA, Pesqueira D, MiguelFraile PS, Ortiz-Rey JA, Antón-Badiola I, et al.: Atypical small glands in prostate needle biopsies. Diagnostic value of clinicopathological parameters. Int $\mathrm{Br} \mathrm{J}$ Urol. 2000; 26: 503-509.

14. Cheville JC, Reznicek MJ, Bostwick DG: The focus of "atypical glands, suspicious for malignancy" in prostatic needle biopsy specimens: incidence, histologic features, and clinical follow-up of cases diagnosed in a community practice. Am J Clin Pathol. 1997; 108: 633-40.

15. Brausi M, Castagnetti G, Dotti A, De Luca G, Olmi R, Cesinaro AM: Immediate radical prostatectomy in patients with atypical small acinar proliferation. Over treatment? J Urol. 2004; 172: 906-9; discussion 908-9.

16. Shepherd D, Keetch DW, Humphrey PA, Smith DS, Stahl D: Repeat biopsy strategy in men with isolated prostatic intraepithelial neoplasia on prostate needle biopsy. J Urol. 1996; 156: 460-2; discussion 462-3.

Received: December 17, 2004 Accepted after revision: March 21, 2005

\section{Correspondence address:}

Dr. Katia Ramos Moreira Leite

Rua Dona Adma Jafet, 91

Sao Paulo, SP, 01308-050, Brazil

Fax: + 5521 231-2249

E-mail: katiaramos@uol.com.br 